Elsevier required licence: (C) <2019>. This manuscript version is made available under the CC-BY-NCND 4.0 license http://creativecommons.org/licenses/by-nc-nd/4.0/

The definitive publisher version is available online at

[https://www.sciencedirect.com/science/article/pii/S0376738819306386?via\%3Dihub] 


\title{
Systematic insight into the short-term and long-term effects of magnetic microparticles and nanoparticles on critical flux in membrane bioreactors
}

\author{
Hongcheng Guo ${ }^{\mathrm{a}, \mathrm{b}, 1}$, Jiajun $\mathrm{Hu}^{\mathrm{b}, 1}$, Jixiang $\mathrm{Li}^{\mathrm{a}, \mathrm{c}, * *}$, Min-tian Gao ${ }^{\mathrm{b}}$, Qiyuan Wang ${ }^{\mathrm{e}}$, Wenshan Guo ${ }^{\mathrm{d}}$, \\ Huu Hao $\mathrm{Ngo}^{\mathrm{d}, *}$ \\ ${ }^{a}$ Shanghai Advanced Research Institute, Chinese Academy of Sciences, Shanghai 201210, China \\ b Shanghai Key Laboratory of Bio-Energy Crops, School of Life Sciences, Shanghai University, Shanghai 200444, China \\ ${ }^{\mathrm{c}}$ University of Chinese Academy of Sciences, Beijing 100049, China \\ ${ }^{\mathrm{d}}$ School of Civil and Environmental Engineering, University of Technology Sydney, Sydney, NWS 2007, Australia \\ ${ }^{\mathrm{e}}$ FuXing Senior High School, Shanghai 200434, China \\ * Corresponding author. \\ ** Corresponding author. Shanghai Advanced Research Institute, Chinese Academy of Sciences, Shanghai 201210, \\ China. \\ E-mail addresses: lijixiang@sari.ac.cn (J. Li), HuuHao.Ngo@uts.edu.au (H.H. Ngo). \\ 1 equal contribution.
}

Keywords: Short-term effect, Long-term effect, Critical flux, Flocculation, Magnetic induced bio-effect

Abstract

This study aims to systematically investigate the short-term and long-term effects of magnetic microparticles (MPs) and nanoparticles (NPs) on critical flux in membrane bioreactors (MBRs). Comparison among six MBRs was carried out with different activated sludge samples. Results showed that the short-term adsorption and flocculation contributed only minimally, however, the long-term magnetic induced bio-effect improved the critical flux by conditioning sludge properties. Additional molecular weight distribution of soluble microbial product (SMP) indicated that long-term magnetic induced bioeffect declined the content of macromolecules ( $>500 \mathrm{kDa}$ and $300-500 \mathrm{kDa}$ ), but promoted the content of small molecules $(<100 \mathrm{kDa}$ ), consequently reduced the free energy of SMP gelling foulants, and further promoted the higher critical flux. Moreover, the magnetic MPs presented the better performance than NPs. This study illustrated that sufficient pre-acclimatization of magnetic activated sludge is significantly necessary to improve the critical flux in MBRs.

\section{Introduction}

Membrane bioreactor (MBR) has been considered as a promising technology for wastewater treatment and reuse [1]. However, membrane fouling is still the major concern of its widespread application [2]. Extensive efforts have been executed to explore the mechanism and mitigation of membrane fouling in MBRs [3]. Chen et al. [4] and Yu et al. [5] proposed a new fouling mechanism based on Flory-Huggins theory, and precisely quantified the interfacial interactions between foulants and membranes. Chen et al. [6] developed a macroporous resin - MBR hybrid system and successfully mitigated the membrane fouling by improving sludge properties.

Magnetic activated sludge (MAS) process, a modified conventional activated sludge process, has displayed distinct advantages for improving bioreactor performance [7]. Considering the responsiveness of magnetic particles to the magnetic field, they can be easily separated, reactivated and reused, thus MAS process is cost-efficient. Recently, it also displayed great potential for mitigating membrane fouling in MBRs
[8]. Wang et al. [9] found that adding magnetic particle could effectively alleviate membrane fouling by encouraging microbes to produce less membrane foulants. Liu et al. [8] found that magnetic bio-effect could significantly mitigate membrane fouling by reducing the amounts of macromolecules and pioneer bacteria from bulk sludge. Although MAS process has been extensively investigated for improving biomass growth and pollutants removal [10], the information on mitigating fouling in MBRs is still limited. So far, systematic insight into mitigating fouling by MAS process is necessary in order to develop effective fouling control strategies.

Critical flux $\left(J_{c}\right)$, as an index to characterize the amount of fouling, was proposed by Field et al. [11] and widely used to assess the fouling trend and to effectively control fouling. In this study, the objective was to systematically investigate the short-term and long-term effects of magnetic microparticles (MPs) and nanoparticles (NPs) on critical flux in MBRs. For this purpose, six MBRs were compared with different activated sludge samples. Statistical analysis was used to further analyze the correlation between various parameters of sludge mixture and 


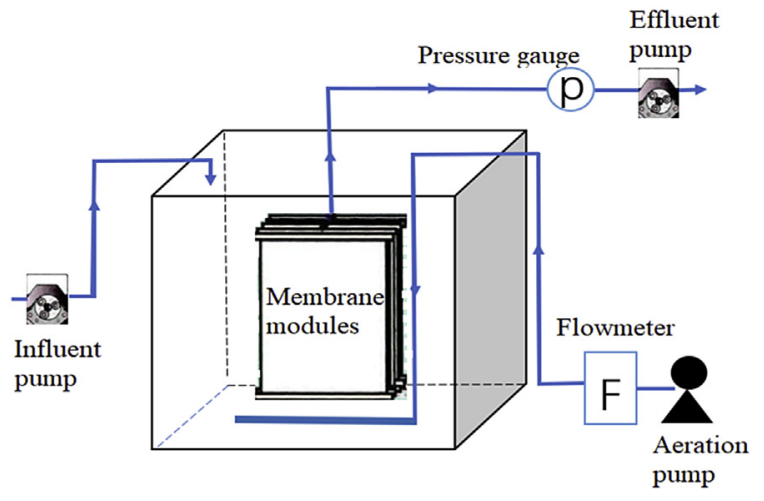

Fig. 1. The schematic diagram of the MBRs.

critical flux. As far as we know, this is the first systematic investigation on the short-term and long-term effects of magnetic MPs and NPs on critical flux in MBRs. It is expected that the information provided in this study will be helpful to explore the mechanism and mitigation of membrane fouling by MAS process in MBRs.
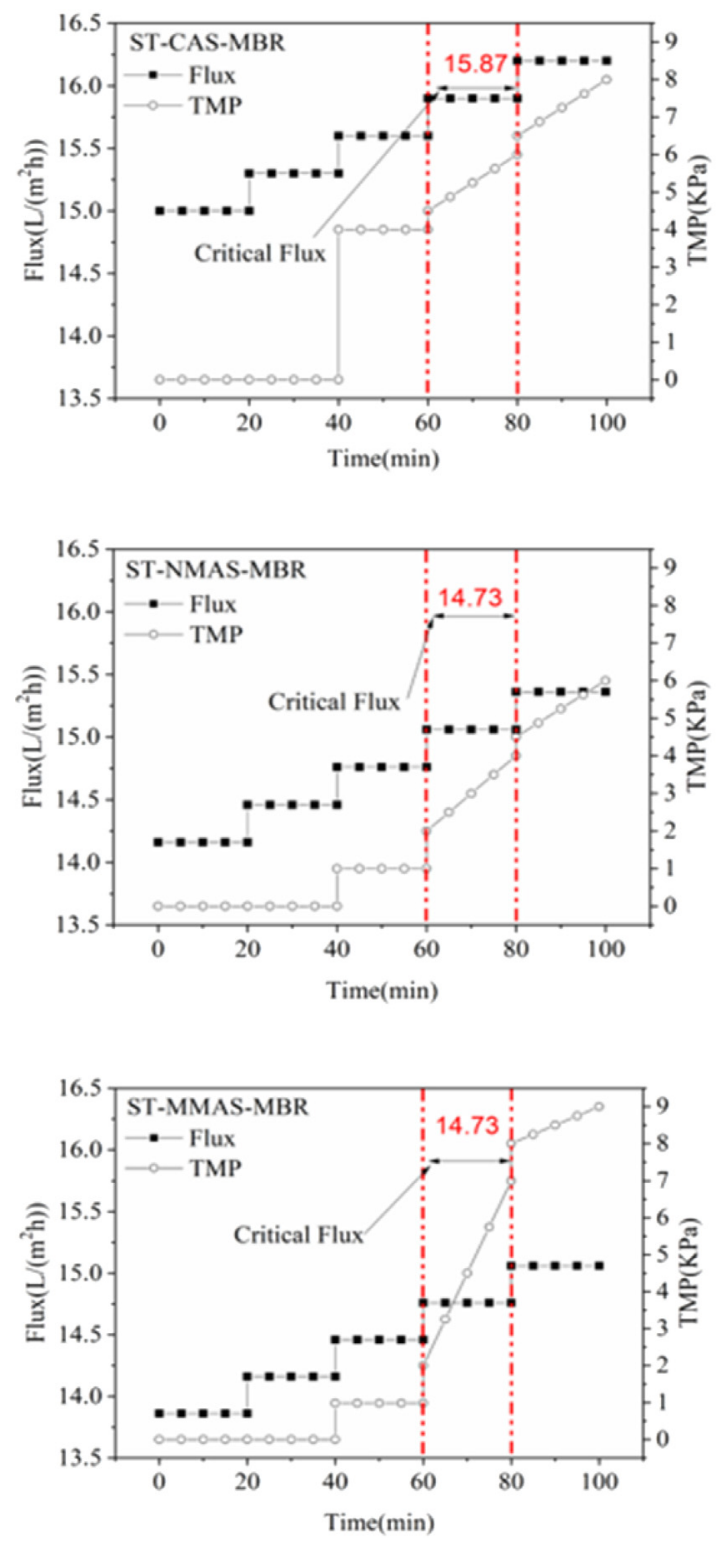

\section{Materials and methods}

\subsection{Setup and the operation of MBRs}

To carry out the experiments, six aerobic MBRs (with a working volume of $4 \mathrm{~L}$ ) were operated. The schematic diagram of the MBRs was provided in Fig. 1. For the short-term effect experiments, three MBRs were operated. One is the control MBR with seeded activated sludge (ST-CAS-MBR), one is the test MBR with seeded activated sludge and $1 \mathrm{~g} / \mathrm{L}$ freshly added magnetic MPs (ST-MMAS-MBR), and another one is the test MBR with seeded activated sludge with $1 \mathrm{~g} / \mathrm{L}$ freshly added magnetic NPs (ST-NMAS-MBR). Both of the freshly added magnetic particles were mixed with seeded activated sludge for $60 \mathrm{~min}$. For the long-term effect experiments, three MBRs were operated with acclimated activated sludge without magnetic particle (LT-CAS-MBR), acclimated activated sludge with $1 \mathrm{~g} / \mathrm{L}$ added magnetic MPs (LT-MMASMBR), and acclimated activated sludge with $1 \mathrm{~g} / \mathrm{L}$ added magnetic NPs (LT-NMAS-MBR), respectively. All the acclimated activated sludge samples were cultivated for more than 60 days.
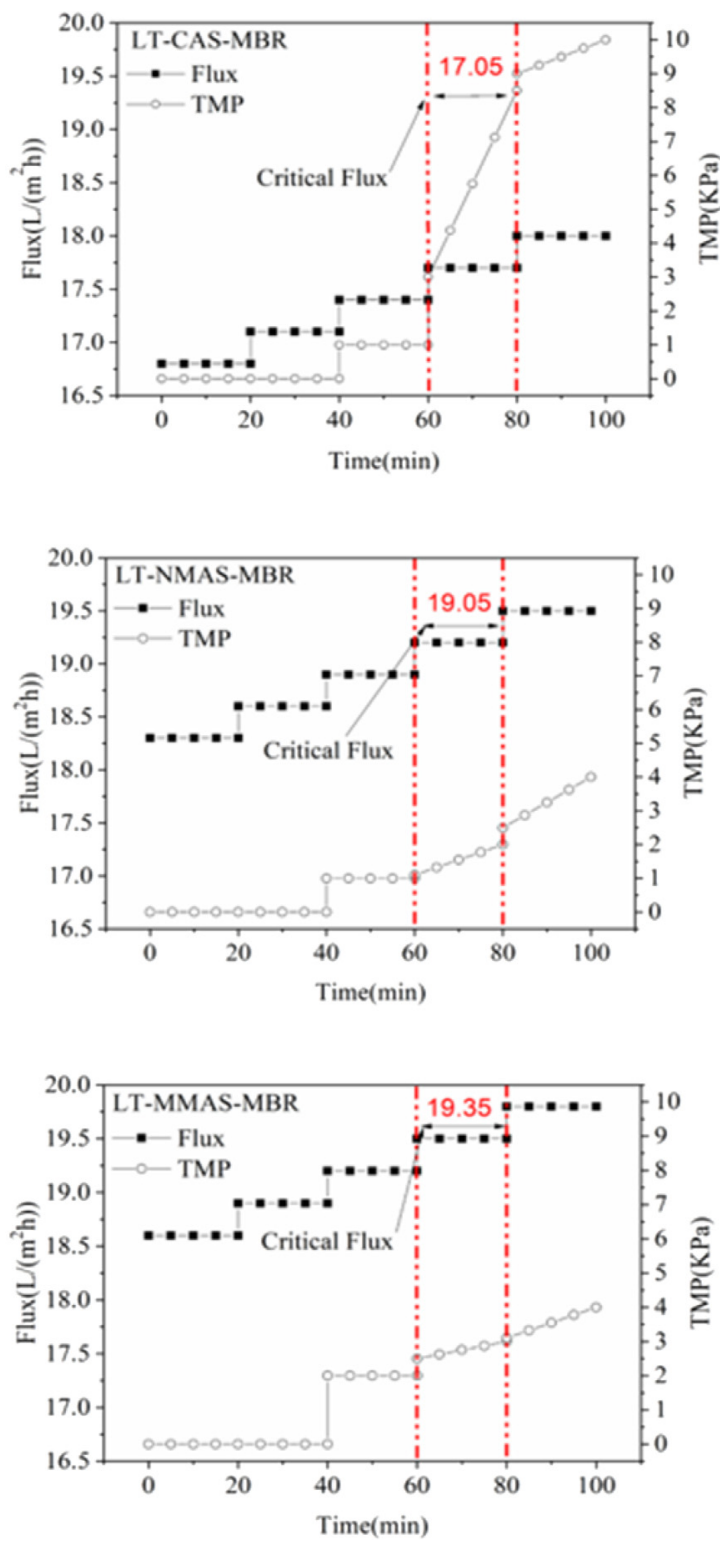

Fig. 2. The profiles of critical flux and TMP from the six MBRs. 
Table 1

The sludge properties from the six MBRs.

\begin{tabular}{|c|c|c|c|c|c|c|c|c|c|c|}
\hline Samples & $\operatorname{CST}(\mathrm{s})$ & $\mathrm{SMP}_{\mathrm{c}}(\mathrm{mg} / \mathrm{g})$ & $\mathrm{SMP}_{\mathrm{p}}(\mathrm{mg} / \mathrm{g})$ & $\mathrm{EPS}_{\mathrm{c}}(\mathrm{mg} / \mathrm{g})$ & $\mathrm{EPS}_{\mathrm{p}}(\mathrm{mg} / \mathrm{g})$ & PSD $(\mu \mathrm{m})$ & TTC (mg/g) & $\mathrm{SVI}_{30}(\mathrm{~mL} / \mathrm{g})$ & $\Delta \mathrm{R}_{30}\left(10^{8} / \mathrm{m} \cdot \mathrm{h}\right)$ & $J_{c} \mathrm{~L} /\left(\mathrm{m}^{2} \cdot \mathrm{h}\right)$ \\
\hline LM & 15.90 & $3.11 \pm 0.16$ & $0.66 \pm 0.03$ & $18.07 \pm 0.90$ & $24.01 \pm 1.20$ & 227.00 & 3.83 & 83.33 & 0.50 & 19.35 \\
\hline LN & 16.20 & $4.53 \pm 0.23$ & $2.08 \pm 0.10$ & $28.68 \pm 1.43$ & $36.90 \pm 1.84$ & 124.00 & 1.79 & 74.18 & 0.60 & 19.05 \\
\hline LC & 12.10 & $4.36 \pm 0.22$ & $0.85 \pm 0.04$ & $20.45 \pm 1.02$ & $22.79 \pm 1.14$ & 136.00 & 3.03 & 84.49 & 1.09 & 17.64 \\
\hline SM & 14.80 & $9.66 \pm 0.48$ & $1.29 \pm 0.06$ & $20.15 \pm 1.00$ & $27.48 \pm 1.39$ & 56.00 & 1.12 & 128.13 & 2.53 & 14.73 \\
\hline SN & 12.90 & $9.09 \pm 0.46$ & $1.38 \pm 0.07$ & $25.53 \pm 1.28$ & $32.37 \pm 1.62$ & 56.00 & 1.84 & 87.50 & 2.47 & 14.73 \\
\hline SC & 20.60 & $9.75 \pm 0.49$ & $1.85 \pm 0.09$ & $25.84 \pm 1.27$ & $32.57 \pm 1.62$ & 56.00 & 1.26 & 171.88 & 2.35 & 15.87 \\
\hline
\end{tabular}

* LM: LT-MMAS-MBR, LN: LT-NMAS-MBR, LC: LT-CAS-MBR, SM: ST-MMAS-MBR, SN: ST-NMAS-MBR, SC: ST-CAS-MBR.

\subsection{Determination of critical flux}

Critical flux was measured via the stepwise flux method [12]. The step length and step height were chosen to be $20 \mathrm{~min}$ and $0.3 \mathrm{~L} /\left(\mathrm{m}^{2} \cdot \mathrm{h}\right)$, respectively.

\subsection{Analytical methods}

The mixed liquor suspended solids (MLSS) was tested according to Chinese NEPA standard methods. The particle size distribution (PSD) of sludge samples was measured using a particle size analyzer (Malvern 2000, United Kingdom). The molecular weight (MW) was tested by a gel permeation chromatography (GPC) (Shimadzu LC-20AD, Japan). Activated sludge activity was characterized by triphenyltetrazolium chloride (TTC) method. Soluble microbial product (SMP) and extracellular polymeric substances (EPS) were extracted based on a modified thermal method, and quantified as carbohydrate and protein [8].

\subsection{Magnetic particles adsorption batch tests}

Magnetic particles adsorption batch tests were performed with extracted membrane foulant solution (i.e. SMP) and seeded activated sludge for the model adsorption and practical adsorption tests, respectively.

\subsection{Statistical analysis}

Statistical analysis software (SPSS 17.0) was used to analyze the correlation between various parameters of sludge mixture and critical flux.

\section{Results and discussion}

\subsection{The profiles of critical flux and TMP in MBRs}

Six aerobic MBRs were operated to evaluate the effects of magnetic particles on critical flux. The profiles were provided in Fig. 2. It can be seen that the short-term effect caused the decline of critical flux, whereas the long-term effect increased the critical flux. The critical fluxes of the ST-MMAS-MBR and ST-NMAS-MBR both declined from $15.87 \mathrm{~L} /\left(\mathrm{m}^{2} \cdot \mathrm{h}\right)$ of ST-CAS-MBR to $14.73 \mathrm{~L} /\left(\mathrm{m}^{2} \cdot \mathrm{h}\right)$. The freshly added magnetic particles may serve as inorganic foulants on membrane, and consequently resulted in the critical flux decline. On the contrary, the critical fluxes of the LT-MMAS-MBR and LT-NMAS-MBR increased from $17.64 \mathrm{~L} /\left(\mathrm{m}^{2} \cdot \mathrm{h}\right)$ of LT-CAS-MBR to $19.35 \mathrm{~L} /\left(\mathrm{m}^{2} \cdot \mathrm{h}\right)$ and $19.05 \mathrm{~L} /\left(\mathrm{m}^{2} \cdot \mathrm{h}\right)$, respectively. Moreover, magnetic MPs displayed the better performance than NPs, which may be caused by the long-term damage to microbes from nano-effect. Therefore, it can be inferred that the short-term adsorption and flocculation of magnetic particles contributed minimally to critical flux enhancement whereas the long-term magnetic induced bio-effect improved the critical flux by conditioning sludge properties. In other words, these results illustrated that the pre-acclimatization of magnetic activated sludge is essential to improve the critical flux in MBRs. In practical applications, the enhancement of critical flux can effectively reduce the amount of membrane modules and in turn decrease the capital investment. From the viewpoint of operating cost, the higher critical flux contributed to reducing the amount of aeration and chemical consumption for membrane cleaning. Thus the present work is of great importance for MBRs. These results also suggest that the shortterm magnetic adsorption and flocculation should be enhanced by functional magnetic composite materials, such as magnetic adsorbents and magnetic coagulants.

\subsection{Effect of magnetic particles on sludge properties}

Sludge properties have been widely considered to have significant impact on critical flux in MBRs [13]. The addition of magnetic particles may cause the variations of sludge properties from physiochemical and biological aspects, and finally may affect the critical flux. In order to assess their effect, the sludge properties from the six MBRs were compared and summarized in Table 1. Statistical analysis was further used to reveal the correlation between sludge properties and critical flux.

It can be seen from Table 1 that sludge properties of short-term MBRs were quite different from those of long-term MBRs, especially the significant parameters associated with critical flux, such as SMPc, SMPp, PSD, $\mathrm{SVI}_{30}, \Delta \mathrm{R}_{30}$, and TTC. These results provided direct evidences that the short-term adsorption and flocculation of magnetic particles did not well condition sludge properties and contributed little effect on critical flux enhancement. The magnetic induced bio-effect improved sludge properties, and consequently enhanced the critical flux. Therefore, it can be concluded that the magnetic activated sludge process was a feasible and practical method for flux improvement in MBRs, and the premise was that there must be a pre-acclimatization step before the normal operation of MBRs.

It should be noted that the CST values of sludge mixture were obviously shortened in the short-term groups, whereas those of long-term groups were prolonged. This implied that the short-term addition of magnetic particles can effectively improve the dewaterability of activated sludge. At the same time $\mathrm{SVI}_{30}$ tests demonstrated that adding magnetic particles by short-term or long-term way both improved the sedimentation property of activated sludge. The lower $\mathrm{SVI}_{30}$ may be resulted from the adsorption of magnetic particles onto activate sludge. PSD analysis showed that sludge mixtures from three short-term MBRs had similar floc size, indicating that adding magnetic particles had little effect on sludge flocculation. That was to say, the short-term flocculation effect contributed minimally to critical flux enhancement.

It was expected that the short-term exposure of magnetic MPs did not affect bacterial activity while the long-term exposure improved the dehydrogenase activity. It was unexpected that the short-term exposure of magnetic NPs stimulated a rapid activation of bacteria whereas the long-term exposure significantly decreased the dehydrogenase activity. $\mathrm{Ni}$ et al. [14] got the opposite conclusion. They found that the sudden short-term exposure of $50 \mathrm{mg} / \mathrm{L}$ magnetic NPs inhabited bacterial activity whereas the long-term exposure enhanced bacterial activity. The contradiction may be aroused by the higher dose of $1 \mathrm{~g} / \mathrm{L}$ magnetic NPs in this study. Therefor the acute toxicity and long-term stability of magnetic NPs in MBRs should be evaluated in the future study. 


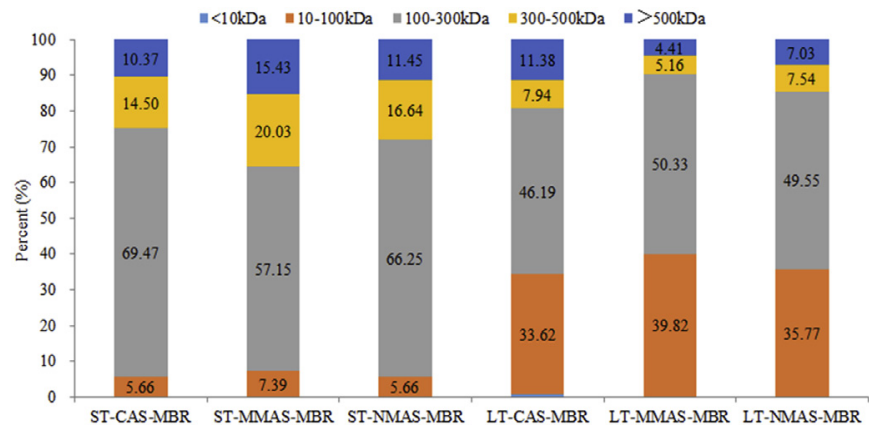

Fig. 3. MW distributions of SMP from the six MBRs.

\subsection{Effect of magnetic particles adsorption on membrane foulants}

To further confirm the adsorption effect of magnetic particles on membrane foulants, batch model adsorption and practical adsorption tests were performed with extracted SMP solution and activated sludge, respectively. Results showed that the practical adsorption presented a little higher capacity than the model adsorption. Maybe there existed partially flocculation of magnetic particles in the practical adsorption with seeded activated sludge. However, the magnetic NPs did not display the expected higher adsorption capacity than MPs on account of there being little affinity. Semblante et al. [15] reported the similar results. These results confirmed that the short-term adsorption effect contributed minimally to critical flux enhancement.

\subsection{Effect of magnetic particles on molecular weight distribution of SMP}

It has been well recognized that SMP generally featured more macromolecules than EPS [16], and Liu et al. [8] confirmed that the addition of magnetic particle in the MBR could significantly reduce the macromolecules of SMP but little impact on EPS. GPC therefor was used to compare the MW distributions of SMP from the six MBRs.

Fig. 3 presented the MW distributions of SMP from the six MBRs. Obviously, the three MBRs from short-term groups showed similar MW distributions, while the three MBRs from long-term groups showed significant differences. In the short-term groups, the proportions of macromolecules (MW > $500 \mathrm{kDa}$ and $300-500 \mathrm{kDa}$ ) from ST-MMASMBR (15.43\% and 20.03\%) and ST-NMAS-MBR (11.45\% and $16.64 \%)$ were larger than those from ST-CAS-MBR (10.37\% and 14.5\%). This may be associated with the critical flux decline in ST-MMAS-MBR and ST-NMAS-MBR compared with ST-CAS-MBR. In the long-term groups, the proportions of macromolecules (MW $>500 \mathrm{kDa}$ and $300-500 \mathrm{kDa}$ ) from LT-MMAS-MBR $(4.41 \%$ and 5.16\%) and LT-NMAS-MBR $(7.03 \%$ and $7.54 \%$ ) both declined compared with those from LT-CAS-MBR $(11.38 \%$ and $7.94 \%)$. On the contrary, the proportions of small molecules $(<100 \mathrm{kDa})$ from the LT-MMAS-MBR $(39.82 \%)$ and LT-NMASMBR (35.77\%) both increased compared with LT-CAS-MBR (33.62\%). Correspondingly, LT-MMAS-MBR and LT-NMAS-MBR displayed higher critical fluxes compared with LT-CAS-MBR. It has been reported that magnetic induced bio-effect could affect the microbes and their metabolism in MBRs, and then affect the membrane fouling [8]. Similarly, macromolecules in MBRs could be successfully transformed into small molecules through the long-term magnetic induced bio-effect, which further promoted the higher critical flux. This could be explained by the latest chemical potential mechanism in Flory-Huggins theory [17-19]. Small molecules fabricated the low cross-linking and low mixing free energy (i.e., chemical potential), which reduced the filtration resistance. Therefore, it can be concluded that the critical flux enhancement should be attributed to the free energy reduction of SMP gelling foulants with small molecules.

\section{Conclusion}

The short-term and long-term effects of magnetic MPs and NPs on critical flux in MBRs were systematically evaluated in this study. It was found that, the short-term adsorption and flocculation contributed minimally whereas the long-term magnetic bio-effect improved the critical flux. In particular, the long-term magnetic bio-effect successfully converted the macromolecules into small molecules, reduced free energy of SMP gelling foulants, and further promoted the higher critical flux. For the practical applications of MBRs, the present work is of importance to reduce the capital investment and operating cost. The study may also provide helpful information for the cultivation and application of MAS process to mitigate membrane fouling and have great potential to develop effective fouling control strategies in MBRs.

\section{Acknowledgements}

The authors appreciate the support of the National Natural Science Foundation of China (51878646), the National Key R\&D Program of China (2017YFE0116300), the Youth Innovation Promotion Association, CAS (2017353).

\section{Appendix A. Supplementary data}

Supplementary data to this article can be found online at https:// doi.org/10.1016/j.memsci.2019.04.015.

\section{References}

[1] S.T. Mu, S. Wang, S. Liang, K. Xiao, H.J. Fan, B.J. Han, C. Liu, X.M. Wang, X. Huang, Effect of the relative degree of foulant "hydrophobicity" on membrane fouling, J. Membr. Sci. 570-571 (2019) 1-8.

[2] J.H. Teng, M.J. Zhang, K.-T. Leung, J.R. Chen, H.C. Hong, H.J. Lin, B.-Q. Liao, A unified thermodynamic mechanism underlying fouling behaviors of soluble microbial products (SMPs) in a membrane bioreactor, Water Res. 149 (2019) 477-487.

[3] S. Modarresi, M.M. Benjamin, Using adsorbent mixtures to mitigate membrane fouling and remove NOM with microgranular adsorptive filtration, J. Membr. Sci. 573 (2019) 528-533.

[4] J.R. Chen, H.J. Lin, L.G. Shen, Y.M. He, M.J. Zhang, B.-Q. Liao, Realization of quantifying interfacial interactions between a randomly rough membrane surface and a foulant particle, Bioresour. Technol. 226 (2017) 220-228.

[5] G.Y. Yu, X. Cai, L.G. Shen, J.R. Chen, H.C. Hong, H.J. Lin, R.J. Li, A novel integrated method for quantification of interfacial interactions between two rough bioparticles, J. Colloid Interface Sci. 516 (2018) 295-303.

[6] W.W. Chen, J. Luo, R.Y. Cao, Y.T. Li, J.R. Liu, Effect of macroporous adsorption resin-membrane bioreactor hybrid system against fouling for municipal wastewater treatment, Bioresour. Technol. 224 (2017) 112-117.

[7] B. Ma, S. Wang, Z. Li, M. Gao, S. Li, L. Guo, Z. She, Y. Zhao, D. Zheng, C. Jin, X. Wang, F. Gao, Magnetic $\mathrm{Fe}_{3} \mathrm{O}_{4}$ nanoparticles induced effects on performance and microbial community of activated sludge from a sequencing batch reactor under long-term exposure, Bioresour. Technol. 225 (2017) 377-385.

[8] Y. Liu, Q. Liu, J. Li, H.H. Ngo, W. Guo, J. Hu, M. Gao, Q. Wang, Y. Hou, Effect of magnetic particle on membrane fouling mitigation and microbial community/ composition in membrane bioreactors (MBRs) for municipal wastewater treatment, Bioresour. Technol. 249 (2018) 377-385.

[9] H. Wang, Z. Chen, J. Miao, Y. Li, A novel approach for mitigation of membrane fouling: concomitant use of flocculant and magnetic particle, Bioresour. Technol. 209 (2016) 318-325.

[10] Y. Liu, J. Li, W. Guo, H.H. Ngo, J. Hu, M.-T. Gao, Use of magnetic particle to effectively improve the performance of sequencing batch reactors (SBRs) in municipal wastewater treatment, Bioresour. Technol. 248 (2018) 135-139.

[11] R.W. Field, D. Wu, J.A. Howell, B.B. Gupta, Critical flux concept for microfiltration fouling, J. Membr. Sci. 100 (1995) 259-272.

[12] T.-T. Nguyen, S. Kook, C. Lee, R.W. Field, I.S. Kima, Critical flux-based membrane fouling control of forward osmosis: behavior, sustainability, and reversibility, J. Membr. Sci. 570-571 (2019) 380-393.

[13] B. Wu, F. Zamani, W. Lim, D. Liao, Y.Y. Wang, Y. Liu, J.W. Chew, A.G. Fane, Effect of mechanical scouring by granular activated carbon (GAC) on membrane fouling mitigation, Desalination 403 (2017) 80-87.

[14] S.-Q. Ni, J. Ni, N. Yang, J. Wang, Effect of magnetic nanoparticles on the performance of activated sludge treatment system, Bioresour. Technol. 143 (2013) 555561.

[15] G.U. Semblante, S.D.R. Tampubolon, S.-J. You, Y.-F. Lin, T.-C. Chang, F.-C. Yen, Fouling reduction in membrane reactor through magnetic particles, J. Membr. Sci. 435 (2013) 62-70.

[16] M. Aslam, A. Charfi, G. Lesage, M. Heran, J. Kim, Membrane bioreactors for 
wastewater treatment: a review of mechanical cleaning by scouring agents to control membrane fouling, Chem. Eng. J. 307 (2017) 897-913.

[17] M.J. Zhang, H.J. Lin, L.G. Shen, B.-Q. Liao, X.L. Wu, R.J. Li, Effect of calcium ions on fouling properties of alginate solution and its mechanisms, J. Membr. Sci. 525 (2017) 320-329.

[18] M.J. Zhang, H.C. Hong, H.J. Lin, L.G. Shen, H.Y. Yu, G.C. Ma, J.R. Chen, B.-Q. Liao, Mechanistic insights into alginate fouling caused by calcium ions based on terahertz time-domain spectra analyses and DFT calculations, Water Res. 129 (2018) 337-346.

[19] J.H. Teng, L. Shen, G.Y. Yu, F.Y. Wang, F.Q. Li, X.L. Zhou, Y.M. He, H.J. Lin, Mechanism analyses of high specific filtration resistance of gel and roles of gel elasticity related with membrane fouling in a membrane bioreactor, Bioresour. Technol. 257 (2018) 39-46. 